\title{
Geschichte der Naturwissenschaften in Aarau
}

\author{
Von Heinz Balmer
}

\section{Das Aufblühen der Naturwissenschaften in Aarau}

Als 1798 das alte Bern unterging, verkündeten die Aargauer die Bildung eines neuen Kantons. Das bisherige Untertanenstädtchen Aarau wurde zur Kantonshauptstadt, ja, im helvetischen Einheitsstaat bis 1803 sogar zum schweizerischen Hauptort Helvetiopolis. Eine oberste Lehranstalt als geistiges Zentrum wurde 1802 auf privater Grundlage eröffnet. Als Lehrer zog man viele Deutsche heran. Ernst August Evers, der erste Rektor, stammte aus Halle. Er war ein klassischer Philologe, dem das Griechische über alles ging. 1813 wurde die Kantonsschule verstaatlicht. Evers, dem nun Zügel angelegt waren, verließ sie 1817. Der Altphilologe Rudolf Rauchenstein wurde 1825 der zweite bedeutende Rektor.

Aarau galt als moderne Stadt, nicht als altmodisch wie Zürich und Bern. Johann Heinrich Zschokke aus Magdeburg ließ sich als Schriftsteller, Heinrich Remigius Sauerländer aus Frankfurt als Verleger in Aarau nieder. Zschokke wurde der schweizerische Voltaire. Der konservative Berner Staatsrechtslehrer Karl Ludwig von Haller fand die liberale Kantonsschule «abscheulich» und nannte die Druckerei Sauerländer ein «Arsenal des Jakobinismus».

Auf der neu aufgebrochenen Erde gediehen die Naturwissenschaften. Pompejus Alexander Bolley aus Heidelberg (1812-1870), ein Burschenschafter, der 1838 als Lehrer an der Kantonsschule Zuflucht fand, verbesserte die Stellung der Naturwissenschaften. Er zog 1855 als Chemiker an das neue Zürcher Polytechnikum, zu dessen Rektor er aufstieg. Der Geologe und Biologe Friedrich Mühlberg und der Physiker August Tuchschmid prägten die folgende Zeit der Kantonsschule. Beide waren glänzende Lehrer. 1896 bezogen sie die mustergültigen Fachabteilungen im Ostflügel des neuen Schulgebäudes. Das Gymnasium war zum Kristallisationspunkt der geistigen Kräfte des Kantons geworden.

1811 entstand die «Naturhistorische Klasse der Gesellschaft für vaterländische Kultur». Zu den acht Gründungsmitgliedern gehörten Heinrich Zschokke, Rektor Evers und Pfarrhelfer Andreas Wanger, ein eifriger Naturaliensammler (1774 bis 1836). Nachdem Zürich 1746, Bern 1786, Genf 1790 eine Naturforschende Gesellschaft erhalten hatten, war jene in Aarau die viertälteste der Schweiz. Sie zählte bis 1820 im Durchschnitt elf Mitglieder. In den vierziger Jahren kam sie fast zum 
Erliegen. Aber seit Bolley anfing, die Vortragstätigkeit zu beleben, nahm sie Aufschwung. Man tagte im Rößli, seit 1862 im Casino, seit 1871 im Kreuz, später in der Kantonsschule. Im Winter gab es Vorträge, im Sommer Lehrausflüge. Abhandlungen wurden gedruckt: einer ersten «Festschrift» von 1869 folgten seit 1878 «Mitteilungen», die Sauerländer verlegte.

Neben die Kantonsschule und die Naturforschende Gesellschaft trat als drittes das Museum für Natur- und Heimatkunde. Von Anfang an hatte die Gesellschaft ein Naturalienkabinett geplant. Albrecht Rengger, Franz Xaver Bronner und Pfarrhelfer Wanger stifteten ihre Mineraliensammlungen. 1866 verkaufte ihr der aus Aarau stammende Bundesrat Friedrich Frey-Herosé seine Sammlung europäischer Vögel. Die 6000 Franken waren gut angelegt. Der in der Sammlung vertretene ausgestorbene Riesenalk (Alca impennis) blieb das wertvollste Tier des Museums. Zuerst stapelte man die Schätze in der alten Kantonsschule auf. Zudem belegte man Räume außerhalb. Als 1872 das Casino einging, wurde es gemietet. Leider erwies es sich als sehr feucht. 1896 konnte ein Teil der Sammlungen in das neue Kantonsschulgebäude einziehen. Aber immer noch fehlte ein zweckmäßiges Museum. Da gründete 1906 der Aarauer Industrielle Hermann Kummler-Sauerländer (1863-1949), der eine Firma für elektrische Leitungen errichtet hatte, ein Initiativkomitee für den Neubau eines naturhistorischen Museums. 1917 löste sich die Platzfrage; 1920 begann der Bau, und 1922 wurde er eröffnet. Der Zementfabrikant Rudolf Zurlinden war sein größter Gönner.

Zwei Kantonsschullehrer wirkten beim Aufbau der Schausammlung als treibende Kräfte. Es waren der Biologe Paul Steinmann und der Chemiker Adolf Hartmann. In der Villa nebenan sind die wissenschaftlichen Sammlungen untergebracht. Der Jurist Dr. h.c. Gottfried Keller, ein Orchideenforscher, schenkte 400 Orchideenaquarelle. Siegfried Schwere, der als Lehrer am Lehrerinnenseminar wirkte, und nach ihm der Botaniker Hans Ulrich Stauffer, der leider früh starb, bemühten sich um das Typenherbar.

\section{Kurzbiographien tragender Persönlichkeiten}

Vorbereitet wurde die Blütezeit durch drei Männer, die noch im 18. Jahrhundert wurzelten. Der erste und älteste war Vater Johann Rudolf Meyer (1739-1813). Er war der Sohn eines Gerbers in Aarau. Als Seidenbandfabrikant gelangte er zu Reichtum. Angeregt von Franz Ludwig Pfyffers Relief der Urschweiz in Luzern, wollte Meyer ein Relief der Schweiz herstellen lassen. Mit dem Straßburger Ingenieur Johann Heinrich Weiss besuchte er die Alpen, kam nach Engelberg und 


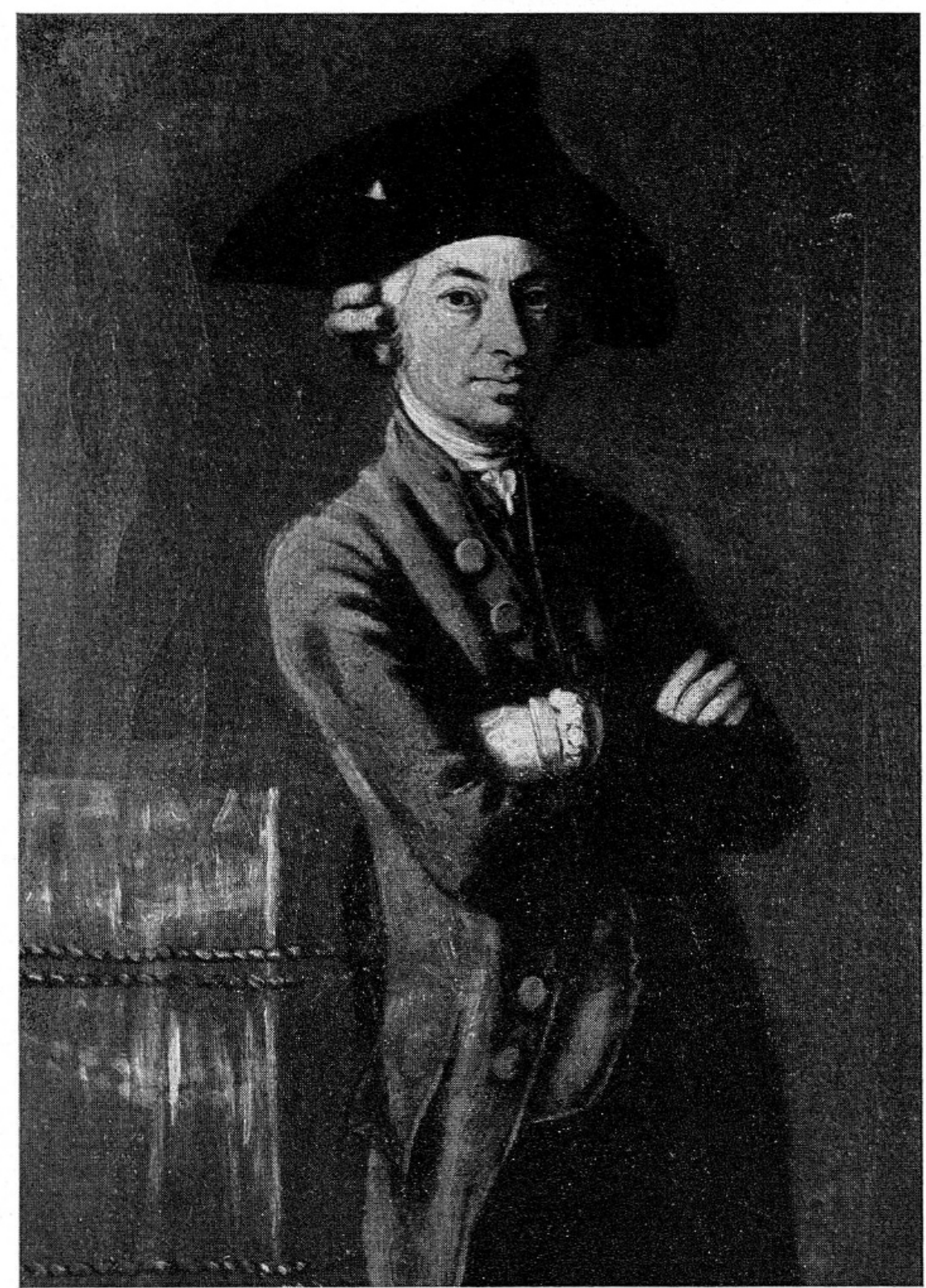

Johann Rudolf Meyer (1739-1813)

bestieg den Titlis. Als Führer begleitete sie der Engelberger Zimmermann Joachim Eugen Müller. Da dieser mit seltener Gebirgskenntnis auf Fehler in ihren Skizzen hinwies, lud Meyer ihn nach Aarau ein und stellte ihn an. Weiss und Müller lernten bei Georg Tralles in Bern das Basismessen und maßen 1789 bei Aarau eine Basis. Von hier aus wurde ein Dreiecksnetz vermessen. Müller stieg mit Schachteln voll Gips auf die Berge und formte nach, was er von oben sah. Meyer wollte 
das Relief in Papiermasse vervielfältigen. Da es nicht gelang, ließ er von Weiss einen Atlas stechen. Die Reliefs von Müller dienten dem Zeichner als hilfreiche Unterlagen. Die 16 Blätter entstanden 1796 bis 1802. Erstmals war das Hochgebirge richtig von oben erfaßt und in Schraffen wiedergegeben.

Auch sonst bewährte sich Meyer als Geniefinder. 1801 hatte er einen Elsässer Reißzeugmacher, Ludwig Esser, nach Aarau kommen lassen. Ihm gab er einen elternlosen Knaben aus Mollis, dessen er sich angenommen hatte, in die Lehre. Es war Jakob Kern, der zum Schöpfer einer der bekanntesten Firmen Aaraus wurde. - Beim Bau seiner Seidenweberei beobachtete Meyer einen besonders tüchtigen Maurer aus dem Tirol; ihn stellte er an für den Vertrieb der Seidenbänder und bildete auch seine Knaben aus. Ein Enkel, Carl Franz Bally, gründete die Schuhfabrik in Schönenwerd. - Meyer hatte auch eigene Söhne; der älteste, Johann Rudolf, der die Fabrik übernahm, gab eine vierbändige Naturlehre heraus und bestieg als erster die Jungfrau.

Die zweite Persönlichkeit war Franz Xaver Bronner (1758-1850). Er war der Sohn eines Ziegeleiarbeiters in Bayern und wurde Mönch, floh aber 1785 in die Schweiz und wurde Notensetzer in einem Zürcher Verlag, dann Redaktor. 1804 kam er als Lehrer an die Kantonsschule Aarau. Da Evers die Anliegen des Realunterrichtes nicht unterstützte, zog Bronner als Physikprofessor nach Kasan an der Wolga. Sieben Jahre später kehrte er gerne zurück. Mit Andreas Wanger gab er 1819 ein Mineralienbuch heraus, und 1829 erschien sein ausführliches Rechenbuch. Mit 72 Jahren begab er sich nicht in den Ruhestand, sondern wurde emsiger Kantonsbibliothekar und Kantonsarchivar. Der 85jährige legte das Werk «Der Kanton Aargau» vor. Da er im Hause Essers wohnte, der neben Reißzeugen gute Barometer herstellte, widmete sich Bronner auch der Meteorologie. «Er lebte thätig und bescheiden. Er strebte nach Wahrheit und Licht. Er liebte Natur und Dichtung», steht auf seinem Grabstein.

Heinrich Zschokke (1771-1848), der dritte Wegbereiter der Naturwissenschaften in Aarau, kam um 1796 in die Schweiz. Als Schriftsteller von unglaublicher Fruchtbarkeit, Aufklärer und liberaler Volksbildner verfaßte er Romane, Dramen, Geschichtswerke, politische, religiöse, forstwirtschaftliche Abhandlungen, Zeitungen und Kalender. Er bewog Sauerländer, 1803 in Aarau eine Buchdruckerei einzurichten, und brachte sie mit seiner Feder zur Blüte. Zschokke wurde viel gelesen, und Sauerländer wurde der Verleger, der durch die Teilnahme am geistigen Aufbau der neuen Schweiz geschichtliche Bedeutung empfing. Die «Aarauer Zeitung» mit Paul Usteri als Inland- und Zschokke als Auslandredaktor, die 1815 bis 1821 erschien, war in ihrer Stellung die Vorläuferin der Neuen Zürcher Zeitung. Aarau wurde durch Zschokke ein Mittelpunkt meteorologischer Forschung. 


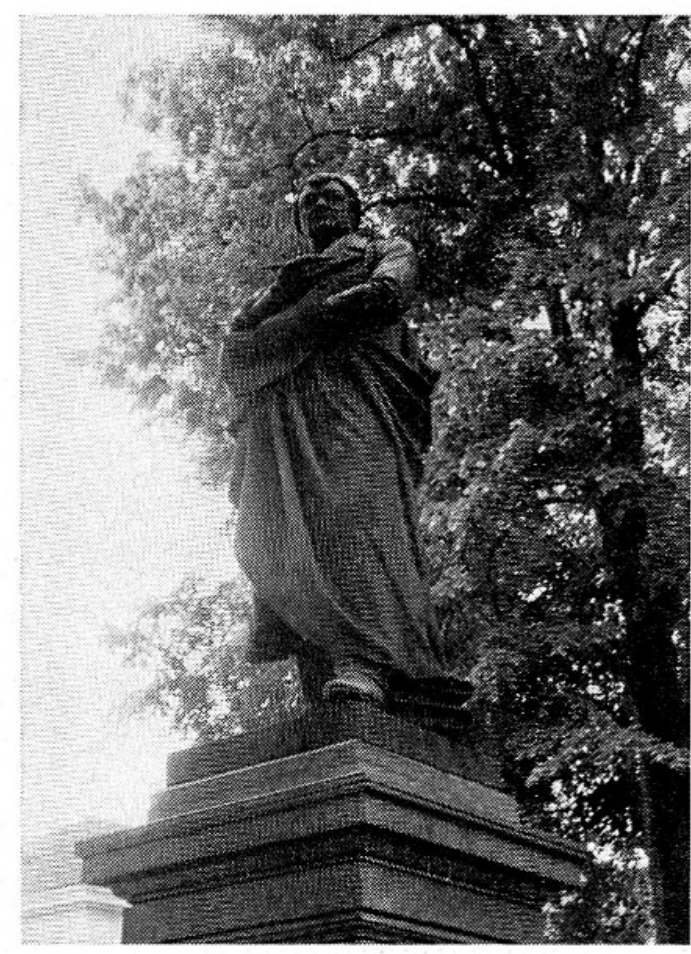

Denkmal Heinrich Zschokkes in Aarau

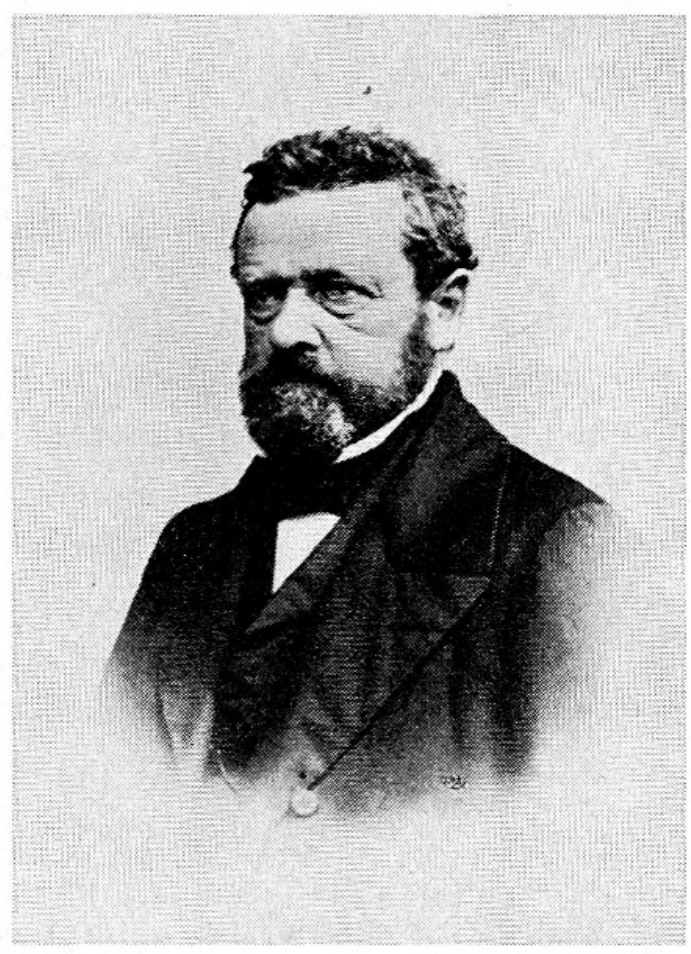

Theodor Zschokke

Arzt und Naturgeschichtslehrer

Die vielseitige Begabung des Vaters spaltete sich auf wie ein Sonnenstrahl im Prisma und fiel in den verschiedenen Farben auf seine zwölf Söhne. Die Witterungskunde pflegte Theodor weiter (1806-1866). Er war Arzt in Aarau, seit 1840 neben Bolley Lehrer an der Kantonsschule. Für die beschreibenden Naturwissenschaften wurde Theodor Zschokke für 25 Jahre der Bannerträger in Aarau.

Die spätere Zeit ist wiederum besonders durch drei Namen gekennzeichnet: Friedrich Mühlberg (1840-1915), Adolf Hartmann (1882-1959) und Paul Steinmann (1888-1953).

Friedrich Mühlbergs Eltern stammten aus Deutschland. Sie betrieben in Aarau eine Stoffdruckerei. Der Sohn studierte in Zürich nur zwei Jahre, aber bei vorzüglichen Lehrern, nämlich beim Geologen Arnold Escher von der Linth, beim Botaniker Oswald Heer und beim Chemiker Pompejus Bolley. Als junger Lehrer an der Kantonsschule Zug entdeckte er Pfahlbauten und wurde 1866 von Erziehungsdirektor Augustin Keller an die Kantonsschule Aarau berufen. Hier entfaltete er sich 45 Jahre. Er ging im Unterricht eigene Wege, um seine Schüler beobachten, denken und schreiben zu lehren. Um die Naturforschende Gesellschaft und um das Museum erwarb er sich größte Verdienste. Er schrieb eine 
große Flora des Aargaus; vor allem aber war er Geologe. Wie es heute die Flüsse tun, so strömten im Aargau einst die Gletscher zusammen. Die von ihnen hinterlassenen Findlinge und Moränen erfaßte Mühlberg als Eiszeitforscher. Der Quellengeologe schuf die Quellenkarte des Aargaus, und der Jurageologe vollendete sieben geologische Karten. Er war ein Held der Arbeit. Unterstützt wurden seine Bestrebungen vom dritten großen Rektor der Kantonsschule, dem Physiker August Tuchschmid (1855-1939). Der Sohn Max Mühlberg wurde Petroleumgeologe.

Ein Schüler und Freund Mühlbergs war Alfred Amsler von Schinznach (1870 bis 1940). Sein Vater war Wirt und Posthalter. Nachdem der Sohn ohne Abschluß Botanik und Geologie studiert hatte, half er daheim. Lieber aber gab er sich Geländestudien hin. An Amanz Greßly erinnern seine Hingabe und Ver-

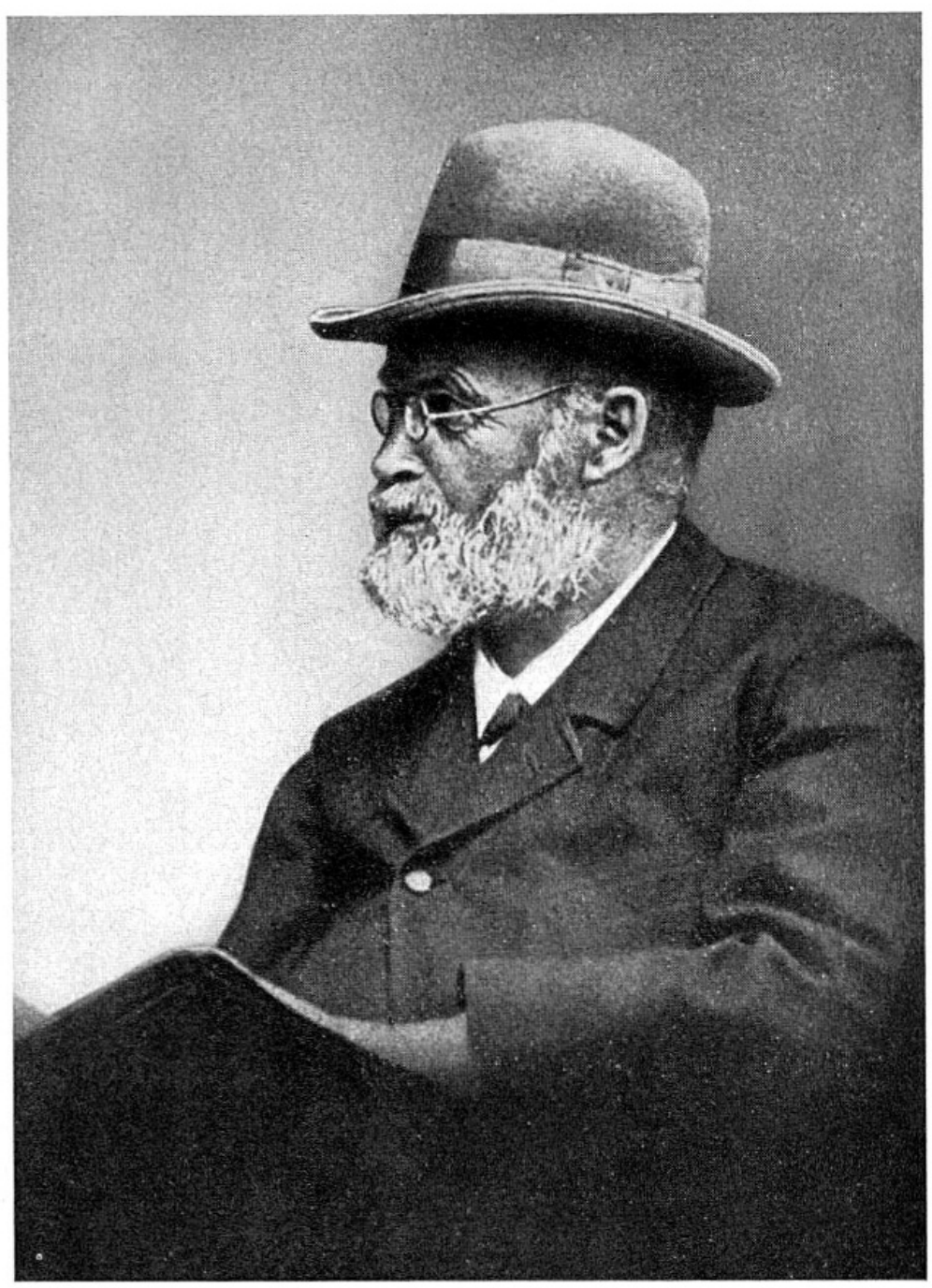


sunkenheit. Ein Lehrbuch der Gesteins- und Bodenkunde, eine Bodenkarte des Aargaus, eine späte Dissertation über die Tektonik des Staffelegg-Gebietes konnten erscheinen. Zeitlebens rang Amsler schwer um seine Existenz. Reich war nur sein Nachlaß.

Adolf Hartmann, ein Bauernsohn aus Schinznach, war seit 1909 Chemielehrer an der Kantonsschule. Der Praktiker half den Gemeinden beim Ausbau ihrer Wasserversorgung; hierin übernahm er das Erbe Mühlbergs. Er kämpfte gegen den Alkohol und warb für Süßmost und Traubensaft. Seiner Feder entflossen ein Chemiebuch und viele Abhandlungen, auch Nachrufe. Außer ihm pflegten die Geschichte der aargauischen Naturwissenschaften Hermann Custer und Mathias Hefti.

Paul Steinmann, als Biologielehrer Hartmanns Kollege an der Kantonsschule, war aus Basel gekommen. Dort hatte er mit einer Untersuchung über die Tierwelt der Gebirgsbäche doktoriert. Er wurde Nachfolger Mühlbergs und verwaltete das Amt 42 Jahre. Die Gaben des Lehrers, Zeichners und Schriftstellers begleiteten ihn. Er verfaßte sowohl naturwissenschaftliche Lehr- und Lesebücher wie wissenschaftliche Arbeiten, vor allem eine Monographie über die Felchen.

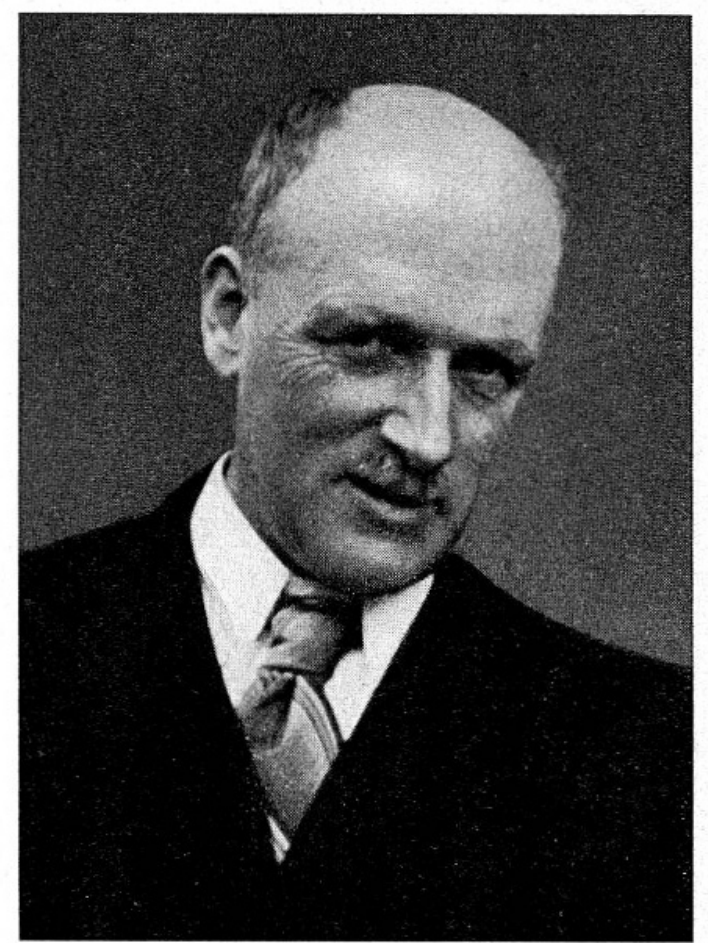

Paul Steinmann (1888-1953)

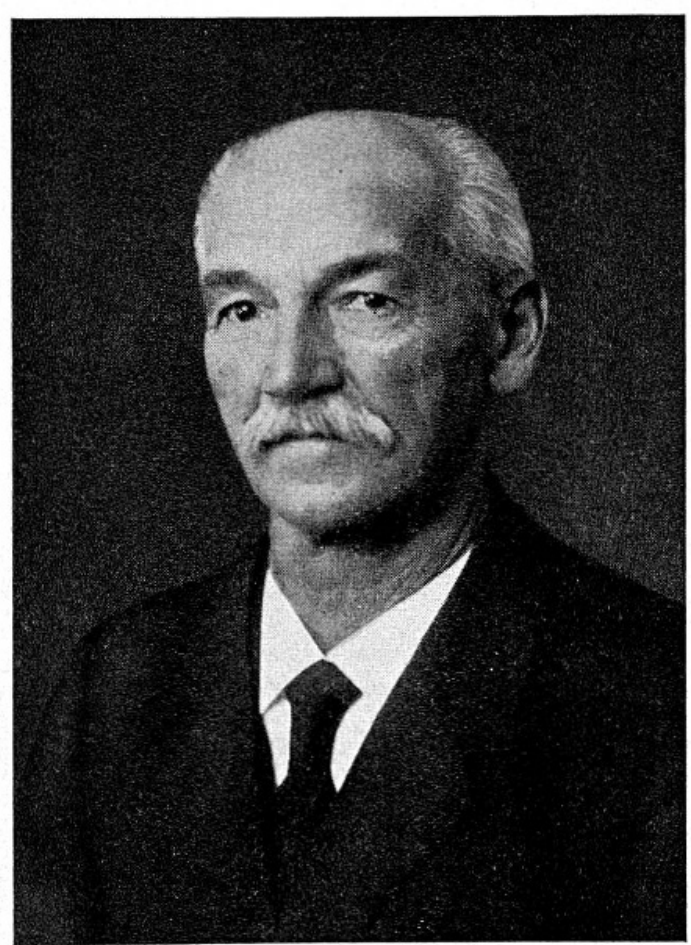

August Tuchschmid (1855-1939) 
Durch seine begeisternden Lehrer wurde Aarau eine Pflanzstätte für naturwissenschaftliche Forscher, die, besonders als Mühlberg-Schüler, von hier auszogen. Nach Zürich wanderten der Zoologe Arnold Lang, der Mineraloge Paul Niggli, der Chemiker Paul Karrer, nach Basel der Zoologe Friedrich Zschokke, der Geologe Carl Schmidt und der Chemiker Arthur Stoll. Aus der Kantonsschule ging auch Alfred Vogt hervor. Als Augenarzt in Aarau untersuchte er mit August Tuchschmid die Durchlässigkeit des Auges für die verschiedenen Strahlen. Er erkannte die Ultrarotstrahlen als Ursache des Gießer- und Glasbläserstars und erfand eine Schutzbrille. 1918 wurde er Professor in Basel, 1923 in Zürich. Als Diagnostiker, Operateur und Vorkämpfer der Spaltlampenmikroskopie erlangte er Weltruf. Ein anderer Zögling, Johann Müller, wurde Professor der Botanik in Genf. Dieser große Systematiker und Erforscher der Wolfsmilchgewächse nannte sich in Anhänglichkeit an seine Heimat Müller Argoviensis. Karl Moser, der Architekt, genannt «Kirchen-Moser», baute nicht nur die Kantonsschule Aarau mit dem kleinen Türmchen, sondern auch die Universität Zürich mit dem großen Turm.

Zu dumm für die Kantonsschule war Alfred Gysi. Er verließ sie vorzeitig und erlernte in Amerika den Zahnarztberuf. Später wurde er Professor der Zahnheilkunde in Zürich und zweifacher Ehrendoktor wegen seiner Forschungen über die Zahnformen und Kaubewegungen.

Nachdem Albert Einstein 1895 die Aufnahmeprüfung an das Zürcher Polytechnikum nicht bestanden hatte, besuchte er einen Winter hindurch die oberste Klasse der Aarauer Kantonsschule. Er wohnte im nahen Rößligut in der Familie seines Geschichtslehrers Jost Winteler, der besonders Germanist und Ornithologe war. Heute trägt das Haus eine Gedenktafel, da das glückliche halbe Jahr den Grund zu Einsteins weiterer Ausbildung legte.

So hat Aarau in die Nähe und in die Ferne gewirkt. Denn was ein Gemeinwesen sich selbst abgewinnt, ist zur Strahlungskraft berufen.

\section{Quellenhinweise}

Sigel

Argovia = Jahresschrift der Historischen Gesellschaft des Kantons Aargau (seit 1860)

Mitt. ANG = Mitteilungen der Aargauischen Naturforschenden Gesellschaft (seit 1878)

Verh. SNG = Verhandlungen der Schweizerischen Naturforschenden Gesellschaft (seit 1823) 


\section{a) Übersicht}

- Mathias Hefti, Zur Geschichte der naturkundlichen Erforschung des Aargaus. Mitt. ANG 24 (1953) 249-286.

\section{b) Zur Aargauischen Kantonsschule}

- Theodor Müller-Wolfer, Die Aargauische Kantonsschule in den vergangenen 150 Jahren, Aarau 1952. 136 S.

c) Zur Aargauischen Naturforschenden Gesellschaft

- Hermann Custer, Bericht über die Thätigkeit der aargauischen naturforschenden Gesellschaft während der Jahre 1863 bis und mit 1877. Mitt. ANG 1 (1878) V-XIV.

- Adolf Hartmann, Geschichte der Aargauischen Naturforschenden Gesellschaft. Mitt. ANG 12 (1911) VIII-XXXI.

- Karl Bäschlin, Aus der Geschichte der Aargauischen Naturforschenden Gesellschaft. Mitt. ANG 26 (1961) 197-211.

- Fanny Custer, Über die ersten Jahresversammlungen der Schweiz. Naturforschenden Gesellschaft in Aarau. Mitt. ANG 17 (1925) 187-194.

d) Zum Aargauischen Museum für Natur- und Heimatkunde

- Friedrich Mühlberg, Das Aargauische Naturhistorische Museum. Mitt. ANG 12 (1911) CXI-CXXVIII.

- Aargauisches Museum für Natur- und Heimatkunde in Aarau. Separatabdruck aus einer Feuilleton-Serie im «Aargauer Tagblatt» 1922. 35 S.

- Adolf Hartmann, Hermann Kummler-Sauerländer und Paul Steinmann, Die Errichtung des Aargauischen Museums für Natur- und Heimatkunde. Mitt. ANG 16 (1923) VII-XLII.

- Siegfried Schwere, Das Typen- und Aargauer-Herbarium im Heimatmuseum. Mitt. ANG 19 (1932) 81-98.

- Rolf Zschokke, Orchideenwerk und Orchideen-Aquarellsammlung von Dr. Gottfried Keller, Aarau. Mitt. ANG 28 (1971) 231-233.

- Eugen Widmer, Verzeichnis des im Aargauischen Museum für Natur- und Heimatkunde in Aarau aufbewahrten wissenschaftlichen Nachlasses der drei Aargauer Geologen Friedrich Mühlberg, Alfred Amsler und Max Mühlberg. Mitt. ANG 28 (1971) 297-308. 
e) Personengeschichte: Sammelbiographien

- Lebensbilder aus dem Aargau 1803-1953. Argovia 65 (1953) 1-488. Darin u. a. Johann Rudolf Meyer, Albrecht Rengger, Heinrich Zschokke, Heinrich Remigius Sauerländer, Ernst August Evers, Augustin Keller, Friedrich FreyHerosé, Rudolf Rauchenstein, Franz Xaver Bronner, Friedrich Mühlberg, Arnold Lang, Alfred Vogt, Paul Niggli.

- Otto Mittler und Georg Boner (Hrsg.), Biographisches Lexikon des Aargaus 1803-1957 (= Argovia 68/69, 1958). 936 S. Kein anderer Kanton der Schweiz besitzt ein ebenso umfassendes Personenlexikon.

f) Personengeschichte: Einzelbiographien (nach dem Abc)

Amsler:

- Alfred Amsler (1870-1940). Mitt. ANG 21 (1943) LI-LX.

Bally:

- Wilhelm Sulser, Carl Franz Bally (1821-1899). In: Schweizer Pioniere der Wirtschaft und Technik, Band 2, Zürich 1955, S. 27-33.

Bolley:

- Pompejus Alexander Bolley (1812-1870). Verh. SNG 1871, S. 265-268.

Bronner (1758-1850):

- Hermann Brunnhofer und Ernst Ludwig Rochholz, Aus Franz Xaver Bronner's Tagebuch einer Reise nach Kasan. Argovia 12 (1881) 87-94.

- Hans Radspieler, Franz Xaver Bronner. Leben und Werk 1794 bis 1850. Argovia 77/78 (1967) 5-199.

- Aargauische Bibliographien und Repertorien I: Hans Radspieler, Franz Xaver Bronner. Hrsg. von der Kantonsbibliothek und vom Staatsarchiv des Kantons Aargau, Aarau 1964. 31 S.

Custer:

- Hermann Custer (1823-1893). Verh. SNG 1893, S. 193-202.

Frey-Herosé (1801-1873):

- Friedrich Frey-Herosé. Aus der handschriftlich hinterlassenen Autobiographie. Argovia 13 (1882) 1-100.

Gysi:

- Alfred Gysi (1865-1957). Mitt. ANG 25 (1958) 232-234.

Hartmann:

- Adolf Hartmann (1882-1959). Mitt. ANG 26 (1961) 240-256.

- Adolf Hartmann. Verh. SNG 1960, S. 218-221. 


\section{Hefti:}

- Mathias Hefti (1894-1961). Mitt. ANG 27 (1966) 261-266.

Karrer:

- Paul Karrer (1889-1971). Verh. SNG 1971, S. 253-255.

Keller, A.:

- Arnold Keller, Augustin Keller, 1805-1883, Aarau 1922. 521 S.

Keller, G.:

- Dr. h.c. Gottfried Keller (1873-1945). Mitt. ANG 22 (1945) 50 f.

\section{Kummler:}

- Hermann Kummler-Sauerländer (1863-1949). Mitt. ANG 23 (1950) 60-64.

- Hermann Kummler-Sauerländer. Verh. SNG 1949, S. 403 f.

\section{Lang:}

- Arnold Lang (1855-1914). Verh. SNG 1915, S. 1-31.

- Aus dem Leben und Wirken von Arnold Lang, Jena 1916. 285 S.

Meyer (1739-1813):

- Ernst August Evers, Vater Johann Rudolf Meyer, Bürger von Aarau. Eine Denkschrift. Aarau 1815. 118 S.

- Rudolf Wolf, Biographien zur Kulturgeschichte der Schweiz, 4 Bände, Zürich 1858-1862. In Band 2: Johann Rudolf Meyer, S. 231-248.

Mühlberg, F.:

- Fritz Mühlberg (1840-1915). Verh. SNG 1915, S. 112-156.

Mühlberg, M.:

- Max Mühlberg (1873-1947). Mitt. ANG 23 (1950) 49-55.

- Max Mühlberg. Verh. SNG 1947, S. 260-264.

Niggli:

- Paul Niggli (1888-1953). Mitt. ANG 25 (1958) 220-224.

- Paul Niggli. Verh. SNG 1953, S. 345-351.

Sauerländer, H.R. (1776-1847):

- Hans Sauerländer, Heinz Sauerländer und Charles Bornet, Hundertfünfzig Jahre Haus Sauerländer in Aarau, Aarau 1957. 119 S.

Schmidt:

- Carl Schmidt (1862-1923). Mitt. ANG 16 (1923) 149-154.

- Carl Schmidt. Verh. SNG 1923, S. 44-54. 
Schwere:

- Siegfried Schwere (1864-1936). Mitt. ANG 20 (1937) 33-37.

Stauffer:

- Hans Ulrich Stauffer (1929-1965). Mitt. ANG 27 (1966) 279-286.

Steinmann:

- Paul Steinmann (1888-1953). Mitt. ANG 25 (1958) 224-228.

- Paul Steinmann. Verh. SNG 1954, S. 355-361.

Tuchschmid:

- August Tuchschmid (1855-1939). Mitt. ANG 21 (1943) XLVIII-L.

Vogt:

- Alfred Vogt (1879-1943). Mitt. ANG 22 (1945) 29-36.

- Alfred Vogt. Verh. SNG 1944, S. 350-355.

Zschokke:

- Heinrich Zschokke (1771-1848). Verh. SNG 1848, S. 154-163.

- Heinrich Zschokke, Eine Selbstschau. Ausgabe: Schweizer Bücherfreunde, Zürich 1939. $248 \mathrm{~S}$.

- Paul Schaffroth, Heinrich Zschokke als Politiker und Publizist während der Restauration und Regeneration. Argovia 61 (1949) 5-203.

- Richard Feller und Edgar Bonjour, Geschichtsschreibung der Schweiz, 2 Bände, Basel/Stuttgart 1962. In Band 2: Heinrich Zschokke, S. 720-723, mit Literaturhinweisen.

\section{Zurlinden:}

- Rudolf Zurlinden-Richner (1851-1932). Mitt. ANG 19 (1932) 49 f.

- Hans Rudolf Schmid, Rudolf Zurlinden, 1851-1932. In: Schweizer Pioniere der Wirtschaft und Technik, Band 13, S. 67-92.

g) Zu Meyers Atlas und Müllers Reliefs

- Rudolf Wolf, Geschichte der Vermessungen in der Schweiz, Zürich 1879. Darin S. 124-126: Johann Heinrich Weiss und Joachim Eugen Müller.

- Walter Blumer, Bibliographie der Gesamtkarten der Schweiz von Anfang bis 1802, Bern 1957. Darin S. 107-110: Der Atlas von Johann Rudolf Meyer.

- Franz Odermatt, Joachim Eugen Müller, 1752-1833. In: Die Alpen 1929, S. 15-26.

- Fritz Gygax, Das topographische Relief in der Schweiz. In: Jahresbericht der Geographischen Gesellschaft von Bern 32 (1935/36) 1-96, mit Anhang von 12 Tafeln. Darin S. 25-30: Joachim Eugen Müller. 


\section{h) Zu Einstein in Aarau}

- Max Flückiger, Albert Einstein in Bern, Bern 1974, S. 24-28.

\section{Dank}

Herr Prof. Dr. Hans Ramser in Aarau schenkte mir Müller-Wolfers Kantonsschulgeschichte. Herr Hans Sauerländer gab mir Hinweise und verhalf mir zu Mitteilungsheften der Aargauischen Naturforschenden Gesellschaft. Herr Albert Steinmann suchte im Verlagsarchiv nach Bildnissen. Jene Meyers und Mühlbergs sind den «Lebensbildern aus dem Aargau», jene Steinmanns und Tuchschmids dem «Biographischen Lexikon des Aargaus» entnommen.

\section{Summary}

In the nineteenth century the town of Aarau became a centre of natural sciences. Many Germans settled there because of her liberal attitude. A classical school, the "Kantonsschule», was founded in 1802. A Society of natural sciences existed since 1811. A Museum for natural history was prepared. The school received a new building in 1896 and the museum in 1922. Leading persons in the beginning of this development were Johann Rudolf Meyer, Franz Xaver Bronner and Heinrich Zschokke. In the following time, Theodor Zschokke and Pompejus Bolley, then Friedrich Mühlberg and August Tuchschmid, afterwards Paul Steinmann and Adolf Hartmann were good teachers of the natural sciences at the Kantonsschule. The long list of their pupils contains several names of important scientists.

Dr. phil. Heinz Balmer

Medizinhistorisches Institut

Rämistraße 71

8006 Zürich 